\title{
The effects of a grape seed extract on ligature induced - periodontitis in rats - an experimental study
}

\author{
TUDOR DIMITRIU1, ZSOFIA DARADICS ${ }^{2}$, SOIMITA SUCIU ${ }^{3}$, ADRIAN CIMPEAN ${ }^{2}$, \\ CORNEL CATOI ${ }^{2}$, GABRIEL ARMENCEA ${ }^{1 *}$, GRIGORE BACIUT ${ }^{1}$, MIHAELA BACIUT ${ }^{1}$ \\ ${ }^{1}$ Iuliu Hatieganu University of Medicine and Pharmacy, Department of Oral and Maxillo- \\ facial Surgery, Cluj-Napoca, Romania \\ ${ }^{2}$ University of Agricultural Sciences and Veterinary Medicine, Department of Pathology, \\ Cluj-Napoca, Romania \\ ${ }^{3}$ Iuliu Hatieganu University of Medicine and Pharmacy, Department of Physiology, Cluj- \\ Napoca, Romania
}

\begin{abstract}
The objective of this study was to evaluate the effects of administering a grape seed extract, rich in polyphenols in an experimental model of ligature induced periodontitis in rats.

Methods: 30 male Wistar rats were divided into Control group, Ligature group and Grape seed extract (GSE) group. Periodontitis was induced by placing a wire ligature around the first mandibular molar for 4 weeks. The animals in the GSE group were administered grape seed extract by gavage. In days 1, 7 and 28 blood samples were taken and in day 28 the hemimandibles were harvested and sent for histological examination. Reduced glutathione (GSH) and malondialdehyde (MDA) were determined in serum and in gingival tissue.

Results: Significantly higher values of MDA were found in serum and periodontium in the Ligature group in comparison to the GSE group. GSE administration resulted in an increase of GSH plasma concentration in the GSE group as compared to the Control and Ligature groups. In the GSE group inflammation and bone involvement were less marked than in the Ligature group.

Conclusions: The grape seed extract proved protective anti-inflammatory and antioxidant effects in a ligature-induced experimental model of periodontitis in rats and also improved the histological status of gingival tissue and bone.
\end{abstract}

Keywords Periodontitis, oxidative stress, grape seed extract, rats.

To cite this article: DIMITRIU T, DARADICS Z, SUCIU S, CIMPEAN A, CATOI C, ARMENCEA G, BACIUT G, BACIUT M. The effects of a grape seed extract on ligature induced - periodontitis in rats - an experimental study. Rom Biotechnol Lett. 2021; 26(1): 2347-2354. DOI: $10.25083 / \mathrm{rbl} / 26.1 / 2347.2354$ 


\section{Introduction}

Periodontitis is a chronic inflammatory disease affecting a significant percentage of population in developed countries (PIHLSTROM \& al [1]). Chronic periodontitis represents a major public health issue, not only because it is the most important cause for tooth loss in adults, but also because recent papers point to its role in development of cardiovascular diseases and in adverse pregnancy outcomes (HARVEY \& al [2], MONEET \& al [3]).

The pathogenesis of periodontal disease is complex. The primary etiologic determinants are specific bacteria in the dental plaque biofilm. However, rather than the pathogens themselves, it is the hosts inflammatory response that leads to tissue destruction (CHAPPLE \& al [4], CHAPPLE \& al [5]). Thus, when stimulated by bacterial pathogens, immune host cells produce a gingivomucosal inflammatory response by releasing IL-1, TNF- $\alpha$ and IL-6 (HAJISHENGALLIS \& al [6]). These proinflammatory cytokines induce the expression of other mediators, such as prostaglandins that amplify the inflammatory response and lead to production of lytic enzymes (SILVA \& al [7]). Neutrophils play an important part in the etiology of periodontitis. They are attracted to the inflammatory site and release proteolytic enzymes and reactive oxygen species (ROS) (WANG \& al [8]). Unless balanced by antioxidant substances, ROS produce cellular damage, by attacking proteins, lipids, sugars and nucleic acids (WANG \& al [8]). Oxidative attack on connective tissue results in structural and biochemical alterations of collagen that appears to play a determinant role in pathogenesis of inflammation related diseases (WANG \& al [8]). ROS can be seen as an important pathogenetic mechanism in periodontal disease (ROBERTS $\&$ al [9]). Numerous studies have indicated that periodontitis and oxidative stress are linked. In experimental models, increased levels of biomarkers for tissue damage induced by reactive oxygen species were found in the periodontal tissues and in the plasma of experimental periodontitis in rats (TOMOFUJI \& al [10]). As for humans, in the gingivocrevicular fluid of periodontitis patients high levels of oxidative markers have been reported, as compared to controls. The role of oxidative stress in periodontal disease has been pointed out by compromised antioxidant capacity in patients suffering from periodontitis (CHAPPLE \& al [5]).

This scientific data brought into light the importance of developing strategies to combat the deleterious effects of reactive oxygen reactive species in gingival tissues. Karim and coworkers provided evidence that a superoxidedismutase mimetic causes a substantial reduction in oxidative damage markers and bone destruction in periodontitis (KARIM \& al [11]). It has been reported that S-nitrosoglutathione, a nitric oxide donor with antioxidant and anti-inflammatory properties decreased matrix metalloproteinases (MMPs) and inhibited bone loss in an experimental model of periodontitis (MARTINS \& al [12]).

In delaying or preventing tissue injuries in periodontitis, certain natural compounds with known antiinflammatory and antioxidant properties may be of interest. Studies in experimental models and in humans gave proof that the antioxidant properties of natural products can be attributed to their capacity to scavenge free radicals and to regulate cell signaling pathways and gene expression (WANG \& al [8]).

Plant polyphenols are abundant compounds in daily diet and their consumption has proved to be beneficial in the prevention of atherosclerosis and cancer (BASU \& al [13], FANTINI \& al [14]). Grapes contain a large variety of polyphenols, in both their skin and seeds. Grape intake has proven to inhibit age-related or cadmium - induced lipid peroxidation (SINGH \& al [15]). It has been reported that extracts from grape seeds exert anti-inflammatory effects similar to indomethacin by reducing polymorphonuclear leukocyte infiltration induced by 12-O-tetradecanoylphorbol 13-acetate and by inhibiting Cox-2 (ÖZDEN $\&$ al [16]). Data on the beneficial effects in oral diseases are few. Daily supplementation of diet with resveratrol reduced the levels of IL- 6 but did not change the TNF- $\alpha$ and total antioxidant capacity of the organism and increased secretion of anti-inflammatory cytokines. (JAVID \& al [17], BUNTE \& al [18]). Resveratrol also attenuated the virulence of Porphyromonas gingivalis by reducing the expression of virulence factor genes such as fimbriae (type II and IV) and proteinases (kgp and $\operatorname{rgpA}$ ), thus decreasing biofilm formation and inflammation of the periodontium (KUGAJI \& al [19]).

The aim of this study was to estimate the effects of a red grapes seed extract in an experimental model of ligature induced periodontitis in rats.

\section{Materials and Methods}

\section{Reagents}

O-phtalaldehyde, 2-thiobarbituric acid, trichloroacetic acid, monobasic potassium phosphate, Bradford and Folin-Ciocalteu reagents were obtained from SigmaAldrich Chemicals GmbH (Darmstadt, Germany). Absolute ethanol, methanol and n-butanol were purchased from Chimopar (Bucharest, Romania). EDTA-Na $\mathrm{Na}_{2}$ was obtained from Merck KGaA (Darmstadt, Germany). Gallic acid was 
obtained from Fluka (Buchs, Switzerland). All chemicals and reagents were of high-grade purity.

A hydroethanolic extract from grape seeds (Vitis vinifera L), variety Burgund Mare (Recas, Romania) was prepared, as previously described, by mixing $1: 10 \mathrm{w} / \mathrm{v}$ finely powdered dried seeds and water/ethanol 50/50 (v/v) and by subsequently concentrating the solution 10 fold in vacuo (POSTESCU \& al [20]). The product was characterized by its total polyphenolic content, assessed by the Folin-Ciocalteu colorimetric reaction and expressed in equivalents (Eq) galic acid (GA) per unit of volume. In previously published data procyanidin $\mathrm{B}$, catechin hydrate, epigallocatechin, epicatechin and gallic acid were identified as main compounds by high-performance liquid chromatography (HPLC-MS) analysis (PERDESCHREPLER \& al [21]).

\section{Animals and induction of periodontitis}

A total of 30 male Albino Wistar rats, 6 months old, weighing $350 \pm 25 \mathrm{~g}$, were used in this experiment. The animals, 5 per cage, were kept at room temperature $23 \pm \pm 0.5^{\circ} \mathrm{C}$ and a $12 / 12$ hours light/dark cycle. The animals received standard diet (Cantacuzino Institute, Bucharest, Romania) and water ad libitum and were let to adapt for 1 week before beginning the experiment.

Ligature placement: The animals were anaesthetized using a mixture of ketamine $(90 \mathrm{mg} / \mathrm{kg}$ b.w.) and xylazine byo $(10 \mathrm{mg} / \mathrm{kg} \mathrm{b.w})$ injected intraperitoneally. For the ligature, 0.5 thick sterile orthodontic wire was placed around the cervix of the first lower left molar and knotted towards the mesial side of the tooth. The wire was situated subgingivally on the buccal, lingual and distal sides of the tooth and supragingivally on the mesial side. This was considered day 0 of the experiment. The ligature was kept in place for 28 days.

The animal care and experimental protocol has been conducted according to local guidelines for animal research and has been approved by the Ethics Committee of "Iuliu Hatieganu" University of Medicine and Pharmacy, Cluj-Napoca, Romania, authorization number 377/16.10.2018.

\section{Experimental groups}

The animals were randomly divided into 3 groups of 10 rats each. Control group and Ligature group: animals received $0.5 \mathrm{ml}$ of saline solution by gavage every 3 days. GSE group: the animals were ligatured and were given red grapes seed extract every 3 days by gavage. The administered dose, based on previous findings, was of $50 \mathrm{mg} \mathrm{GA} / \mathrm{kg}$ body weight.

Tissue samples collection: in days 1, 7 and 28 blood samples were drawn from the ophthalmic venous plexus.
Plasma was separated by centrifugation and frozen at $-180^{\circ} \mathrm{C}$ until biochemical measurements were performed. On the $28^{\text {th }}$ day all the animals were sacrificed using an anesthesia overdose and the first molar regions where the ligature had been placed were resected en bloc and sent for biochemical measurements and histological examination.

\section{Biochemical Assays}

Biochemical analysis was performed in both plasma and periodontal tissues. Tissue fragments were homogenized using a Polytron homogenizer (Brinkman Kinematica, Luzern, Switzerland) for 3 minutes on ice in phosphate buffered saline ( $\mathrm{pH} 7.4)$ added at a ratio of 1:4 (w/v). The cytosolic fraction was obtained by centrifugation for 5 minutes at $3000 \mathrm{~g}$ and $4^{\circ} \mathrm{C}$. Protein concentration in this fraction was measured by the Bradford method.

Malondialdehyde (MDA) was determined as an indicator of lipid peroxidation using a fluorimetric method with 2-thiobarbituric acid. Briefly, to plasma and tissue homogenates, a $10 \mathrm{mM}$ 2-thiobarbituric acid in $75 \mathrm{mM} \mathrm{K} \mathrm{HPO}_{4}$, pH 3 solution was added. The samples were heated in a boiling water bath for 60 minutes and after cooling, the reaction products were extracted in n-butanol. MDA was measured in the organic phase using a synchronous spectrofluorimetric technique (excitation at $534 \mathrm{~nm}$, emission at $548 \mathrm{~nm}$ ), on a Perkin Elmer spectrofluorimeter (Perkin Elmer Inc., Waltham, Massachusets).

Reduced glutathione (GSH) as an indicator of nonenzymic antioxidant defense, was determined by protein precipitation by trichloroacetic acid (10\%) and a spectrofluorimetric assay (350 nm excitation, $420 \mathrm{~nm}$ emission) of the fluorescent derivative obtained by the reaction of the supernatant with a solution of o-phtalaldehyde ( $1 \mathrm{mg} / \mathrm{ml}$ in methanol). GSH concentration was determined using a standard curve. MDA and GSH were expressed as $\mathrm{nmol} / \mathrm{ml}$ in plasma samples and as $\mathrm{nmol} / \mathrm{mg}$ protein in tissue homogenates.

\section{Histological examination}

Slices from periodontal and bone tissues were fixed in $10 \%$ neutral-buffered formaldehyde for 5 days, decalcified, embedded in paraffin and sectioned. Haematoxylin and eosin dyes were used to distinguish the cell types and to characterize the inflammation.

\section{Statistical analysis}

Statistical analysis was performed using MedCalc 18 software. The unpaired two-tailed Student's $t$-test was used to determine the differences between the groups. A threshold of 0.05 was used. The histology evaluation was performed by 2 different observers in different days. 


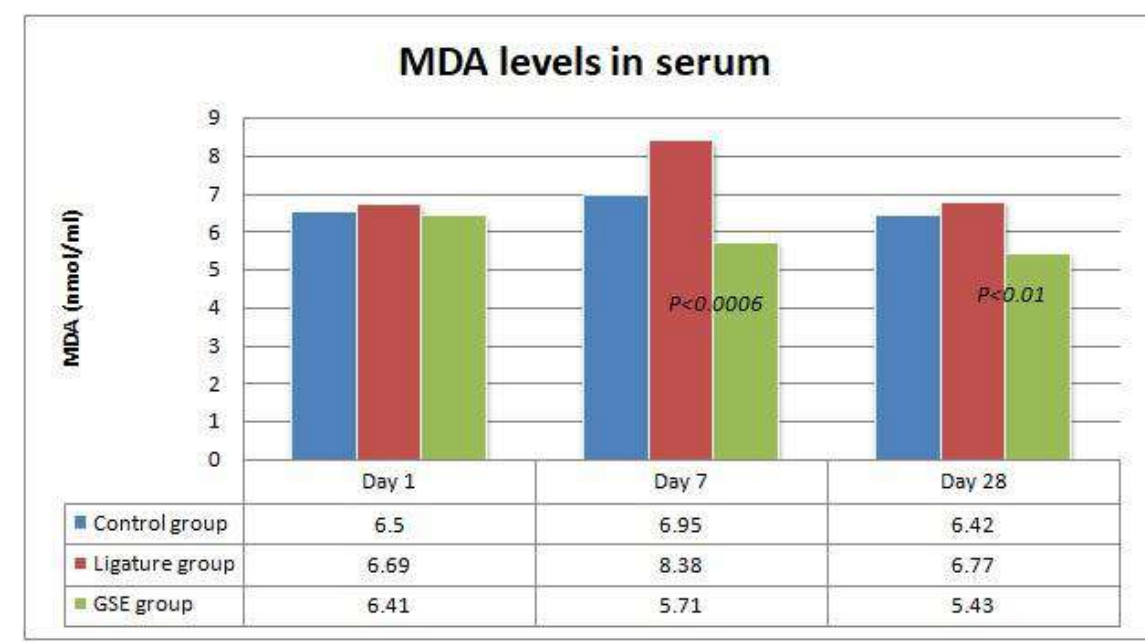

Figure 1. MDA levels in serum

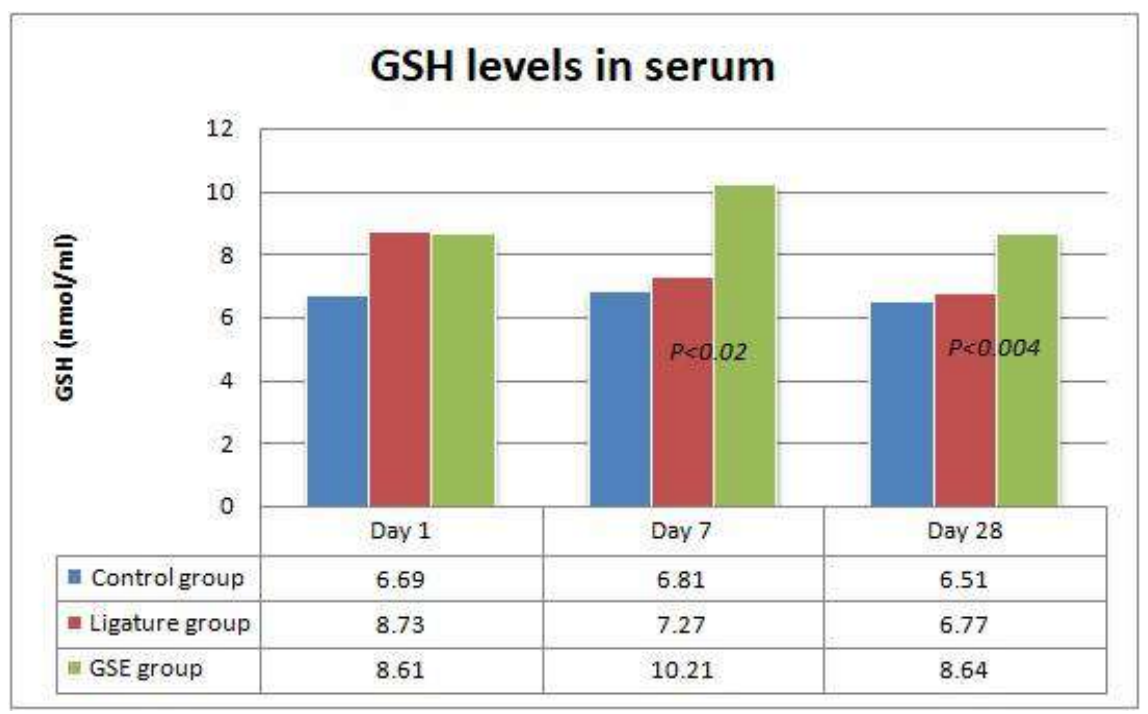

Figure 2. GSH levels in serum

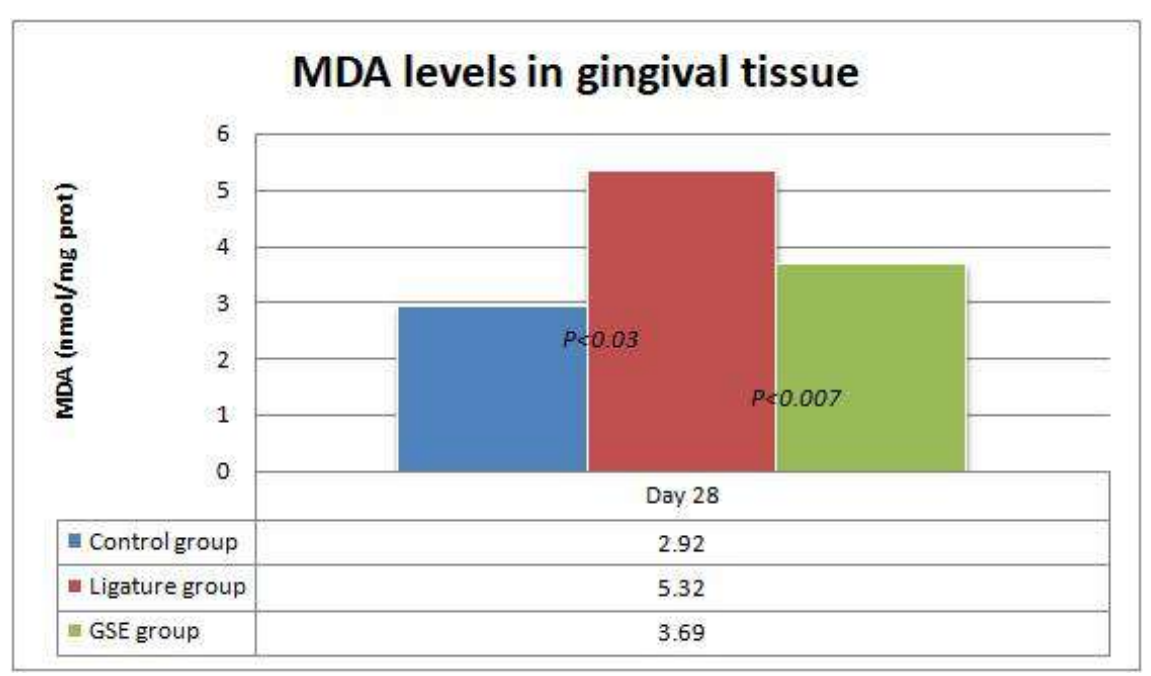

Figure 3. MDA levels in gingival tissue 4 weeks after the placement of the ligature. 


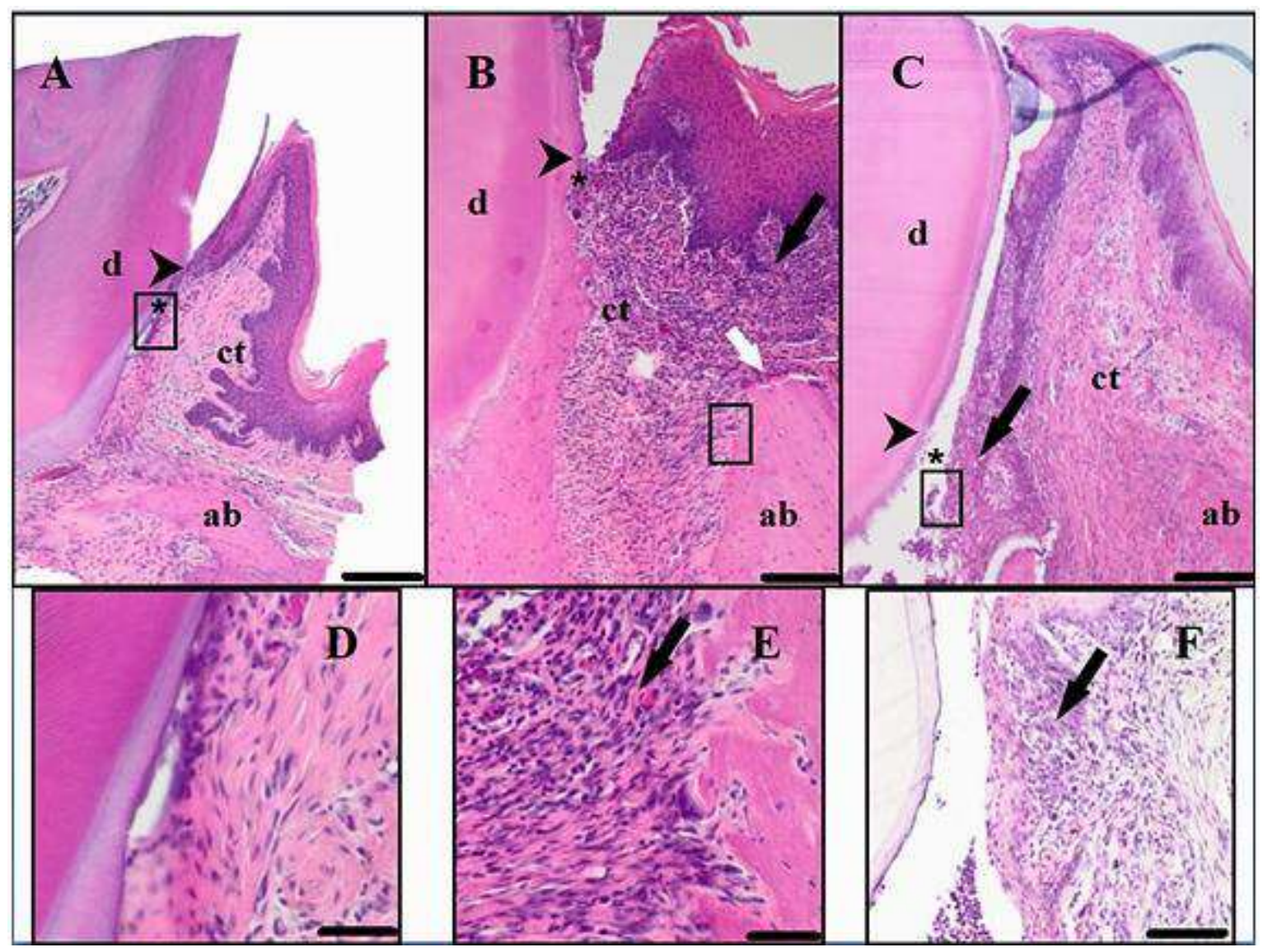

Figure 4. Histological examination of the periodontal tissues stained in Haematoxylin- Eosin after removal of the ligature. No pathologic alterations were identified in the Control group (A, D). The juctional epithelium is situated at the cementoenamel junction. In the Ligatured group apical migration of the junctional epithelium (asterisk), resorption and necrosis of the alveolar bone (white arrow) and inflammatory cells, haematoxylin colored neutrophils (black arrow) were observed $(\mathbf{B}, \mathbf{E})$. In the GSE group the pathologic modifications are less severe. Apical migration of the junctional epithelium (asterisk) and inflammation (black arrow) were present, with no involvement of the alveolar bone. Arrow heads point to the enamel-cement junction. $\mathrm{d}$ - dentin, $\mathrm{ct}$ - connective tissue, $\mathrm{ab}$ - alveolar bone. D, E, F show the magnified area in the black boxes. Scale bar $=200 \mu \mathrm{m}(\mathbf{A}, \mathbf{B}, \mathbf{C})$. Scale bar $=50 \mu \mathrm{m}(\mathbf{D}, \mathbf{E}, \mathbf{F})$.

\section{Results}

\section{Oxidative stress parameters}

Significantly higher values of MDA were found at day 7 in the Ligature group in comparison to the GSE group $(P<0.0006)$. This difference remained until day 28 $(P<0.01)$ (Figure 1).

GSE administration resulted in an increase of GSH plasma concentration as compared to both Control and Ligature group with significant differences between the Ligature group and GSE group at days $7(P<0.02)$ and 28 $(P<0.004)$ (Figure 2).

MDA levels were significantly higher in the periodontal tissues in the Ligature group as opposed to the Control group $(P<0.03)$ and the GSE group $(P<0.007)$ (Figure 3).

\section{Histological examination}

In the Control group no histological modifications occurred (Figure 4. A). The Ligature group presented a high degree of inflammation. Apical migration of the junctional epithelium and bone necrosis were observed (Figure 4. B, E). In the GSE group inflammation and bone involvement were less marked (Figure 4. C, F).

\section{Discussion}

We have studied the effects of a red grape seed extract in a well-established model of ligature-induced periodontitis. Based on the reduction of MDA levels and increase of GSH levels we have found that our product improves non-enzymatic antioxidant defense, reduces lipid peroxidation in plasma and periodontal tissues and 
reduces inflammation and tissue injury related to ligature induced periodontitis.

For some years now the literature indicates a role for radical species as pathogenetic factors in periodontal disease. There have been reports that oxidative stress markers are increased and antioxidant defenses are lower in periodontitis patients in comparison to healthy individuals (TOTHOVE \& al [22], DAHIYA \& al [23]). It has been suggested that severe periodontal disease is independently associated with oxidative stress and reduced antioxidant capacity (D'AIUTO \& al [24]). There are evidences that oxidative stress is linked to inflammation in periodontitis patients in whom peripheral neutrophils are hyperactive in terms of ROS release, even in the absence of exogenous stimulations (D'AIUTO \& al [24], MATTHEWS \& al [25]).

In our study, the placement of a ligature around the first lower molar led to a large amount of neutrophils infiltrating the soft tissues surrounding the tooth. In this group we found also a significant increase of plasma MDA as compared to controls. MDA is frequently used to assess ROS related tissue injuries. In the Ligature group there has been a slight increase of plasma MDA level in the first day after the ligature, which we consider was a consequence of the procedure itself. In day 7 a significant increase of MDA was noticed in the Ligature group. The levels approached initial values and towards the end of the experiment. We attributed this augmentation of plasma lipid peroxidation product to the overproduction of ROS in gingival tissue as we found an increased gingival MDA in ligatured rats as compared to controls. This rationale is supported by the results of Wang et al., who indicated that lipid peroxidation products diffuse from the site of inflammation and can be measured in plasma (WANG \& al [8]). Overproduction of reactive oxygen species is also suggested by the inverse correlation between plasma concentrations of MDA and GSH in ligature group with an increase of MDA and a decrease of GSH levels up to day 7. GSH is a small thiol molecule possessing many biological activities, such as detoxification, stabilization of cell membranes and antioxidant proprieties by scavenging free radicals and reducing peroxides (BAINS \& al [26]). Being a non-enzymic antioxidant, GSH level is consumed in redox reactions. The above mentioned findings denote the presence of inflammation and oxidative stress in the periodontal tissues of ligatured rats.

Histological alterations, such as periodontal tissue detachments and bone necrosis have been associated to inflammation and oxidative stress.

If inflammation and reactive oxygen species play a role in the pathogenesis of periodontal disease, then administration of antioxidants may be beneficial. Some nutritional components can be targeted to this respect. La et al. showed that a grape seed extract inhibited the secretion of MMPs by Actinobacillus actinomycetemcomitans lipopolysaccharide stimulated macrophages and also reduced NFK- $\beta$ levels in patients with periodontitis (LA \& al [27]). Jurikova has shown that polyphenols in berries of blue honeysuckle decreased lipid peroxidation, increased intracellular GHS levels and down-regulated proinflammatory cytokines in human gingival fibroblasts from medically healthy donors (JURIKOVA \& al [28]).

In the present study we have used a grape seed extract in a dosage we previously found to exert both antioxidant and anti-inflammatory effects. Our product diminished the extent of periodontal inflammation and oxidative stress compared to the non-treated ligatured rats. Similar results were reported by Özden (ÖZDEN \& al [16]). This effect can be attributed to the antioxidant effects, as demonstrated by the decreased levels of MDA in this group for the whole duration of the experiment.

Taking into account the steady and substantial increase in plasma GSH levels and also the inverse correlation between MDA and GSH plasma concentration in GSE treated animals, as observed in our study, the beneficial effects of the product we used can be attributed, at least in part, to the increase in GSH levels. Whether this increase is due to an augmentation of its synthesis or to the capacity of polyphenols to prevent its oxidation by quenching ROS, remains a matter of discussion. Zhen has reported that resveratrol, a major component in grapes, exerts antioxidant properties by increasing glutathione biosynthesis in an inflammation experimental model (ZHEN \& al [29]). Resveratrol also reduces alveolar bone loss in experimental periodontitis in rats exposed to cigarette smoke by promoting higher levels of superoxide dismutase (SOD) and lower levels of nicotinamide adenine dinucleotide phosphatase oxidase (NADPH) (CORREA \& al [30]). Systemic treatment with resveratrol reduced rheumatoid factor and anti-citrullinated protein antibodies (ACCPA) levels lowering the bone damage in rats with arthritis and periodontitis (CORREA $\&$ al [31]). Rayyan reported anti-inflammatory effects and reduced probing depth, gingival and plaque index values after applying a grape seed extract gel in periodontal pockets in patients with chronic periodontitis (RAYYAN \& al [32]). Xu showed that quercetin, a plant-derived polyphenol, increases GSH levels by inducing $\gamma$-GCS expression and stimulating GSH synthesis (XU \& al [33]). On the other hand, Afrin has demonstrated that plant polyphenols modulate glutathione redox system in rat proximal colonic mucosa not by increasing its synthesis, but by preventing its oxidation (AFRIN \& al [34]). These discrepancies could be explained by the 
observation that different natural polyphenols exert different effects.

In the present study, because GSH levels in GSE group are significantly higher than in controls, we assume that our product has the ability to augment its synthesis. This might be an important observation, since a number of papers reported decreased levels of GSH within the gingival crevicular fluid in periodontal patients (SAVITA $\&$ al [35]).

Our results suggest a potential therapeutic application of the grape seed extract we have used. Further research is needed to clearly identify the exact component in GSE that acts on oxidative stress and inflammation in ligature-induced periodontitis and the mechanisms of its bioactivity. Future biotechnological development for processing the extract into an available drug has thus become an attractive perspective.

\section{Conclusion}

The grape seed extract from Vitis Vinifera, Burgund Mare variety (Recaş, Romania) proved protective antiinflammatory and antioxidant effects in a ligature-induced experimental model of periodontitis in rats by increasing the non-enzymatic antioxidant capacity, as well as decreasing periodontal tissue and plasma indicators of oxidative stress. It also improved the histological status of gingival tissue and bone.

\section{Acknowledgements}

This study was supported by a $\mathrm{PhD}$ research Grant no. 1680/37/19.01.2017 from the Iuliu Hatieganu University of Medicine and Pharmacy, Cluj-Napoca, Romania.

\section{Conflict of Interest}

The authors have no conflict of interest to declare.

\section{References}

1. PIHLSTROM BL, MICHALOWICZ BS, JOHNSON NW. Periodontal diseases. Lancet, 366, 1809-1820 (2005).

2. HARVEY AS, BRUNO GL. Inflammatory Mechanisms Linking Periodontal Diseases to Cardiovascular Diseases. J Clin Periodontol. 40(0 14): S51-S69 (2013).

3. MONEET W, NAVDEEP S. Relationship between periodontal diseases and preterm birth: Recent epidemiological and biological data. Int J Appl Basic Med Res. 5(1): 2-6 (2015).
4. CHAPPlE ILC, BUSBY M, ClOVER H, MATTHEWS R. Periodontal disease and systemic disease. J R Soc Med. 107(3): 94 (2014).

5. CHAPPLE ILC. Role of free radicals and antioxidants in the pathogenesis of the inflammatory periodontal diseases. Clin Mol Pathol. 49(5): M247-M255 (1996).

6. HAJISHENGALLIS G, LIANG S, PAYNE MA, HASHIM A et al. A Low-Abundance Biofilm Species Orchestrates Inflammatory Periodontal Disease through the Commensal Microbiota and the Complement Pathway. Cell Host Microbe. 10(5): 497-506 (2011).

7. SILVA N, ABUSLEME L, BRAVO D, DUTZAN N et al. Host response mechanisms in periodontal diseases. J Appl Oral Sci. 23(3): 329-355 (2015).

8. WANG Y, ANDRUKHOV O, RAUSCH-FAN X. Oxidative Stress and Antioxidant System in Periodontitis. Front Physiol. 8: 910 (2017).

9. ROBERTS HC, MOSELEY R, SLOAN AJ, YOUDE SJ, WADDINGTON R. Lipopolysaccharide alters decorin and biglycan synthesis in rat alveolar bone osteoblasts: consequences for bone repair during periodontal disease. Eur J Oral Sci. 116(3): 207-216 (2008).

10. TOMOFUJI T, EKUNI D, SANBE T, IRIE K et al. Effects of vitamin $\mathrm{C}$ intake on gingival oxidative stress in rat periodontitis. Free Radic Biol Med. 46(2):163-8 (2009).

11. KARIM S, PRATIBHA PK, KAMATH S, BHAT GS et al. Superoxide dismutase enzyme and thiol antioxidants in gingival crevicular fluid and saliva. Dent Res J. 9(3): 266-272 (2012).

12. MARTINS CS, LEITAO RFC, COSTA DVS, MELO IM et al. Topical HPMC/S-Nitrosoglutathione Solution Decreases Inflammation and Bone Resorption in Experimental Periodontal Disease in Rats. PLoS One. 11(4): e0153716 (2016).

13. BASU A, MASEK E, EBERSOLE JL. Dietary Polyphenols and Periodontitis - A Mini-Review of Literature. Molecules. 23(7): 1786 (2018).

14. FANTINI M, BENVENUTO M, MASUELLI L, FRAJESE GV et al. In Vitro and in Vivo Antitumoral Effects of Combinations of Polyphenols, or Polyphenols and Anticancer Drugs: Perspectives on Cancer Treatment. Int J Mol Sci. 16(5): 9236-9282 (2015).

15. SINGH CK, LIU X, AHMAD N. Resveratrol, in its natural combination in whole grape, for health promotion and disease management. Ann N Y Acad Sci. 1348(1): 150-160 (2015).

16. ÖZDEN FO, SAKALLIOGLU EF, SAKALLIOGLU U, AYAS B et al. Effects of grape seed extract on periodontal disease: an experimental study in rats. J Appl Oral Sci. 25(2): 121-129 (2017). 
17. JAVID AZ, HORMOZNEJAD R, YOUSEFIMANESH HA, HAGHIGHI-ZADEH MH et al. Impact of resveratrol supplementation on inflammatory, antioxidant, and periodontal markers in type 2 diabetic patients with chronic periodontitis. Diabetes Metab Syndr. 13(4):2769-2774 (2019).

18. BUNTE K, HENSEL A, BEIKLER T. Polyphenols in the prevention and treatment of periodontal disease: A systematic review of in vivo, ex vivo and in vitro studies. Fitoterapia. 132:30-39 (2019).

19. KUGAJI MS, KUMBAR VM, PERAM MR, PATL S et al. Effect of Resveratrol on biofilm formation and virulence factor gene expression of Porphyromonas gingivalis in periodontal disease. APMIS. 127(4):187195 (2019).

20. POSTESCU ID, TATOMIR C, CHERECHES G, BRIE I et al. Spectroscopic characterization of grape extracts with potential role in tumor growth inhibition. J Optoel Adv Mater.; 9: 564-567 (2007).

21. PERDE-SCHREPLER M, CHERECHES G, BRIE I, TATOMIR $\mathrm{C}$ et al. Grape seed extract as photochemopreventive agent against UVB-induced skin cancer. J Photochem Photobiol B. 118:16-21 (2013).

22. TOTHOVE L, CELEC P. Oxidative Stress and Antioxidants in the Diagnosis and Therapy of Periodontitis. Front Physiol. 8: 1055 (2017).

23. DAHIYA P, KAMAL R, GUPTA R, BHARDWAJ $\mathrm{R}$ et al. Reactive oxygen species in periodontitis. J Indian Soc Periodontol. 17(4): 411-416 (2013).

24. D'AIUTO F, NIBALI L, PARKAR M, PATEL K et al. Oxidative Stress, Systemic Inflammation, and Severe Periodontitis. J Dent Res. 89(11): 1241-1246 (2010).

25. MATTHEWS JB, WRIGHT HJ, ROBERTS A, COPER PR et al. Hyperactivity and reactivity of peripheral blood neutrophils in chronic periodontitis. Clin Exp Immunol. 147(2): 255-264.

26. BAINS VK, BAINS R. The antioxidant master glutathione and periodontal health. Dent Res J (Isfahan) 2015 Sep-Oct; 12(5): 389-405 (2007).

27. LA VD, BERGERON C, GAFNER S, GRENIER D. Grape seed extract suppresses lipopolysaccharide- induced matrix metalloproteinase (MMP) secretion by macrophages and inhibits human MMP-1 and -9 activities. J Periodontol. 80(11):1875-82 (2009).

28. JURIKOVA T, ROP O, MLCEK J, SOCHOR J et al. Phenolic Profile of Edible Honeysuckle Berries (Genus Lonicera) and Their Biological Effects. Molecules. 17(1): 61-79 (2012).

29. ZHEN L, FAN D, ZHANG Y, CAO X et al. Resveratrol ameliorates experimental periodontitis in diabetic mice through negative regulation of TLR4 signaling. Acta Pharmacol Sin. 36(2): 221228 (2015).

30. CORREA MG, ABSY S, TENENBAUM H, RIBEIRO FV et al. Resveratrol attenuates oxidative stress during experimental periodontitis in rats exposed to cigarette smoke inhalation. J Periodontal Res. 54(3):225-232 (2019).

31. CORREA MG, PIRES PR, RIBEIRO FV, PIMENTEL SP et al. Systemic treatment with resveratrol reduces the progression of experimental periodontitis and arthritis in rats. PLoS One. 13(10):e0204414 (2018).

32. RAYYAN M, TERKAWI T, ABDO H, ABDEL AZIM D et al. Efficacy of grape seed extract gel in the treatment of chronic periodontitis: A randomized clinical study. J Investig Clin Dent. 9(2):e12318 (2018).

33. XU D, HU MJ, WANG YQ, CUI YL. Antioxidant Activities of Quercetin and Its Complexes for Medicinal Application. Molecules. 24(6): 1123 (2019).

34. AFRIN S, GIAMPERI F, GASPARRINI M, FORBES-HERNANDEZ TY et al. Chemopreventive and Therapeutic Effects of Edible Berries: A Focus on Colon Cancer Prevention and Treatment. Molecules. 21(2): 169 (2016).

35. SAVITA AM, SARUN E, ARORA S, KRISHMAN $S$. Evaluation of glutathione level in gingival crevicular fluid in periodontal health, in chronic periodontitis and after nonsurgical periodontal therapy: A clinicobiochemical study. Contemp Clin Dent. 6(2): 206-210 (2015). 